\title{
Processos de aprendizagem, competências aprendidas, funcionamento, compartilhamento e armazenagem de conhecimentos em grupos de pesquisa ${ }^{1}$ Learning processes, competences learned, operation, sharing and storing knowledge
in research groups
}

Catarina Cecília Odelius ${ }^{2}$

Gardênia da Silva Abbad ${ }^{3}$

Pedro Carlos Resende Junior ${ }^{4}$

André de Castro Sena ${ }^{5}$

Caroline Rodrigues Viana ${ }^{6}$

Tatiana Leão Freitas ${ }^{7}$

Tamisia Cristofane Novaes dos Santos ${ }^{8}$

\section{Resumo}

A finalidade deste artigo é descrever o funcionamento, os processos de aprendizagem, armazenagem e compartilhamento de informações em grupos de pesquisa. Para isso, pauta-se por referenciais teóricos oriundos de abordagens cognitivistas e construtivistas. Desenvolvida numa instituição pública de ensino superior, esta pesquisa valeu-se da coleta de dados obtidos por meio de análise documental e de entrevistas semiestruturadas com líderes de 10 grupos. As entrevistas foram registradas em diários de campo e gravadas em áudio. As categorias extraídas dos dados levantados balizaram não apenas as análises de conteúdo, mas também a apresentação de resultados. Foi verificado que os grupos de pesquisa diferem quanto ao objeto e aos métodos de pesquisa, ao tamanho, ao tempo de existência, aos recursos disponíveis, às técnicas e aos equipamentos, o que influencia o desenvolvimento de competências específicas ligadas à realização das pesquisas de cada área. Há semelhanças no que se refere aos processos de aprendizagem, aos conteúdos e às competências genéricas aprendidas por seus integrantes (entre os quais, estudantes de graduação e de pós-graduação). Entre essas competências intelectuais, típicas da produção de conhecimentos, estão: a elaboração de projetos e a coleta, registro e análise de dados. Os resultados indicam também que, quando perguntados sobre o que aprendiam nesses grupos, nove líderes referiram-se especialmente a habilidades relativas à gestão de pessoas, tais como: lidar com diferenças individuais, de ritmo de trabalho, de escolaridade, de experiência profissional, de valores, de crenças e de cultura. A aprendizagem nos grupos ocorre de maneira informal, por meio de conversas entre participantes, e formal, através de leitura dirigida, da participação em eventos científicos e pela realização de pesquisas, sob a supervisão do líder.

\footnotetext{
${ }^{1}$ Uma versão preliminar deste artigo foi apresentada no ENEO de 2010

Artigo submetido em fevereiro de 2010 e aceito para publicação em agosto de 2010
}

2 Doutora em Psicologia pela Universidade de Brasília, Endereço: Universidade de Brasília, Campus Darcy Ribeiro, ala norte, subsolo, módulo 25; CEP 70910900 Brasília, DF. Email:codelius@unb.br

3 Doutora em Psicologia pela Universidade de Brasília. Endereço: SQSW 104, Bloco C, pa. 303, Sudoeste, Brasília, DF, CEP $70670-403$. Email:gardenia.abbad@gmail.com

4 Mestre em Administração pela Universidade de Brasília/PPGA. Doutorando em Administração pela Universidade de Brasília. Endereço: Universidade de Brasília, Campus Darcy Ribeiro, ala norte, subsolo, módulo 25, Brasília, DF, CEP 70910-900. Email:pcrj73@gmail.com

5 Mestre em Administração pela Universidade de Brasília/PPGA; Universidade de Brasília, Endereço: Campus Darcy Ribeiro, ala norte, subsolo, módulo 25; Brasília, DF, CEP 70910-900. Email:andrecsena@gmail.com

6 Mestre em Administração pela Universidade de Brasília/PPGA; Endereço: Universidade de Brasília, Campus Darcy Ribeiro, ala norte, subsolo, módulo 25; Brasília, DF. CEP 70910-900. Email: carolineviana@yahoo.com.br

7 Graduanda em Administração de Empresas pela Universidade de Brasília. Endereço: Universidade de Brasília, Campus Darcy Ribeiro, ICC Norte, S. B1-576, Brasília, DF. CEP: 70910-900. Email: tatiana.If@hotmail.com

8 Graduanda em Administração de Empresas pela Universidade de Brasília. Endereço: Universidade de Brasília, Campus Darcy Ribeiro, ICC Norte, S. B1-576 Brasília, DF. CEP 70910-900. Email: tamisiacristofane@yahoo.com.br 
O compartilhamento de conhecimentos ocorre principalmente durante reuniões para discussão de dados e leitura de artigos, mas também pela utilização de ferramentas da informática por alguns grupos. Os resultados obtidos confirmam a importância da integração de partícipes de diferentes níveis de formação, o que possibilita a aprendizagem, a assimilação de complexas habilidades intelectuais e atitudes, bem como a formação de redes e a busca de reconhecimento e de visibilidade (nacional e internacional) dos conhecimentos produzidos pelo grupo. Esses resultados são discutidos com base nos referenciais teóricos, propondo-se uma agenda de pesquisa sobre a aprendizagem em grupos.

Palavras-chave: processos de aprendizagem; compartilhamento; conhecimento; competências; grupos de pesquisa.

\section{Abstract}

The purpose of this article is to describe the operation, the processes of learning, storing and sharing information in research groups. Therefore, it is guided by cognitive and constructivist theoretical approaches. Developed in a public institution of higher education, this study draws on data collection obtained through documentary analysis and structured interviews with leaders of 10 groups. The interviews were recorded in field diaries and recorded on audio. . The categories obtained from the data collected used as guidelines not only content analysis, but also the presentation of results. The research groups were found to differ on the object and methods of research, the size, time of existence, available resources, techniques and equipment, which influences the development of specific competences of each area. There are similarities in the learning processes, contents and generic competences learned by members (among them, graduate and postgraduate students). These intellectual competences, typical of knowledge production, include: project design, collection and record and data analysis. The results also indicate that, when asked about what they learned in these groups, nine leaders were concerned mainly with the skills related to managing people, such as accept individual differences, velocity of work production, schooling, work experience, values, beliefs and culture. Learning occurs in groups informally, through conversations between participants, and formally, through guided reading, participation in scientific meetings and for practical research under the supervision of the leader. Knowledge sharing occurs mainly during meetings to discuss data and reading articles, but also by using computing tools in some groups. The results confirm the importance of participants' integration from different levels of training and graduation, which enables learning and assimilation of complex intellectual skills and attitudes, as well as providing network building and searching for recognition and visibility (national and international) of knowledge produced by the group. These results are discussed based on theoretical frameworks, proposing an agenda for research into learning in groups.

Key words: learning processes, sharing; knowledge; competences; research groups. 


\section{Introdução}

Estudos relativos à criação de conhecimentos e à aprendizagem em organizações tem sido apontados por vários autores como necessários para que se possa compreender não apenas o modo como esses processos ocorrem e os aspectos que os influenciam (ex.: AKGÜN; LYNN; REILLY, 2002; NONAKA; KROGH; VOEPEL, 2006; ORTENBLAD, 2002), mas também a relação e o processo de transposição da aprendizagem do nível individual para o da organização, em contextos específicos (ARGOTE, 2005; FRIEDMAN; LIPSHITZ; POPPER, 2005). A aquisição, o acúmulo e o uso de conhecimentos também são indicados por vários autores como objeto de estudo (AKGÜN; LYNN; REILLY, 2002).

Entre os aspectos que influenciam esses processos, os autores mencionados destacam o contexto, a atuação de líderes, as relações estabelecidas entre os membros de uma equipe e os aspectos culturais. Em consonância com essas demandas, o estudo em universidades - espaço reconhecido como produtor de conhecimento -, e, mais especificamente, em grupos de pesquisa, tem sido apontado como relevante por vários autores (ACEDO et al, 2006; HOLLIS, 2001, MAUTHNER; DOUCET, 2008), visando compreender os motivos pelos quais os pesquisadores colaboram entre si e o papel e o impacto dessa colaboração na qualidade e na quantidade de artigos publicados, bem como na formação de grupos sociais.

Mauthner e Doucet (2008) destacam o estímulo (até mesmo pressão) verificado nas universidades para que sejam formados grupos de pesquisas multidisciplinares e multi-institucionais, ao mesmo tempo em que ressaltam que a produção de conhecimento por tais grupos demanda um processo dinâmico e complexo.

Visando contribuir para essa discussão - pois no Brasil os órgãos de fomento ao ensino e à pesquisa também têm incentivado a formação de grupos de pesquisa e o estabelecimento de parcerias entre instituições -, este estudo foi realizado com 10 líderes de grupos de pesquisa atuantes numa universidade federal. Os objetivos são descrever a constituição e o funcionamento desses grupos, os processos de aprendizagem, a produção de conhecimento, a disseminação e o armazenamento de informações, bem como as competências individuais, desenvolvidas pelos líderes e pelos estudantes, em função de suas pesquisas. O referencial teórico abrange grupo de pesquisa, aprendizagem em organizações, competências e seus componentes, conhecimentos, habilidades e atitudes.

\section{Grupos de pesquisa: objetivos, funcionamento, requisitos e competências}

A atuação de pesquisadores de modo colaborativo e em equipe tem sido incentivada por órgãos de fomento à pesquisa. Além disso, como já foi destacado, atualmente, há muito estímulo e até pressão nas universidades para que sejam formados grupos de pesquisa multidisciplinares e multi-institucionais (MAUTHNER; DOUCET, 2008). No Brasil, o CNPq, entre inúmeras atividades, organiza e disponibiliza informações sobre grupos de pesquisa em um diretório acessível a toda a sociedade. O intuito é facilitar o intercâmbio e a troca de informações na comunidade acadêmica e científica, preservando a memória da atividade científico-tecnológica no país. De acordo com informações contidas no sítio oficial do CNPq (2010), na página correspondente ao Diretório de Grupos de Pesquisa, grupo de pesquisa é definido como: 
um conjunto de indivíduos organizados hierarquicamente em torno de uma ou, eventualmente, duas lideranças, cujo fundamento organizador dessa hierarquia é a experiência, o destaque e a liderança no terreno científico ou tecnológico, havendo envolvimento profissional e permanente com a atividade de pesquisa, e cujo trabalho se organiza em torno de linhas comuns de pesquisa e que, em algum grau, compartilha instalações e equipamentos.

Conforme Borges-Andrade (2003), uma linha de pesquisa pode ser definida como as orientações teóricas e metodológicas que determinam o rumo da investigação em um dado contexto ou realidade, considerados os limites do campo específico do conhecimento em que se insere o estudo. As linhas de pesquisa representam temas aglutinadores de estudos científicos fundamentados em determinada tradição investigativa, de onde se originam projetos cujos resultados mantêm afinidades entre si. Nesse contexto, um projeto de pesquisa consiste numa investigação com início e final definidos, focada em objetivos específicos, visando à obtenção de resultados de causa e efeito ou à colocação de fatos novos em evidência.

O conceito de grupo de pesquisa corresponde a um grupo de pesquisadores, docentes, estudantes e pessoal de apoio técnico, os quais podem organizar-se em torno de linhas de pesquisa. As interações entre os integrantes desses grupos respeitam uma hierarquia na qual são valorizadas a experiência e a competência técnicocientífica do(s) líder(es). Esse conjunto de pessoas compartilha recursos, informações e instalações físicas a fim de gerar conhecimento científico por meio de processos colaborativos de pesquisa. $\mathrm{O}$ grupo aplica métodos de coleta, análise e interpretação de dados e divulga resultados de pesquisa. Na quase totalidade dos casos, os grupos são compostos pelo docente-pesquisador e por seus estudantes de pós-graduação e/ou graduação. Para um grupo de pesquisa obter registro no $\mathrm{CNPq}$, entre outras exigências, faz-se necessário que o líder do grupo seja:

- bolsista de produtividade do $\mathrm{CNPq}$, pesquisador vinculado a (pelo menos) um programa de pós-graduação stricto sensu;

- doutor ou mestre com projeto de pesquisa (ou de extensão) aprovado nos últimos cinco anos na instituição;

- formalmente indicado por departamento acadêmico (ou pela coordenação de curso) para a constituição de grupo emergente com a finalidade específica de pesquisa ligada a essa unidade acadêmica.

Apesar da atuação em grupos de pesquisa ser considerada importante para a produção de conhecimentos, foram localizados poucos estudos sobre processos de aprendizagem e de produção de conhecimento em grupos desse tipo. A literatura revisada por Du (2009) indica que a ação colaborativa em grupos de pesquisa leva à melhoria das práticas, a novas oportunidades para a reflexão, à redução do isolamento de professores e à adoção de múltiplas perspectivas na solução de problemas. Já Gladney et al (2003) observam uma mudança no padrão de realização da pesquisa quando da presença de equipes multidisciplinares. Embora essa abordagem multidisciplinar possa melhorar o mérito científico e a aplicabilidade dos resultados da investigação (ERLEN, 1997), simultaneamente, cria a necessidade de decisões colaborativas. 
Grupos de pesquisa podem ser considerados ambientes propícios à aquisição e à disseminação interna e externa de habilidades intelectuais complexas como: planejamento de pesquisa, análise e revisão da literatura sobre os temas pesquisados, aplicação de diferentes procedimentos de coleta e análise de dados, redação de textos científicos e coordenação de atividades de pesquisa do grupo. Os conhecimentos declarativos e procedimentais relacionados aos temas e métodos de produção de conhecimentos são, ao que parece, adquiridos por estudantes em disciplinas oferecidas pela universidade. Os mais complexos, relativos à pesquisa, são adquiridos pela participação em grupos de pesquisa. Além de habilidades intelectuais, a aprendizagem em organizações e em grupos de pesquisa, resulta na adoção de novos valores, crenças e atitudes, entre as quais está o respeito à diversidade humana e a disposição para trabalhar de modo coordenado e cooperativo. Os resultados da pesquisa de Odelius e Sena (2009) mostram claramente a emergência de novas atitudes individuais, em decorrência da participação em grupos de pesquisa.

Entre os estudos com objetivo de investigar o desenvolvimento de competências para a realização de atividades de pesquisa, destaca-se o de Cassell et al (2009), que realizaram 45 entrevistas com indivíduos interessados em desenvolver ou aprimorar métodos qualitativos para a pesquisa em administração. A amostra abrangeu: editores de periódicos do campo da administração, pesquisadores do campo que trabalham com métodos quantitativos, pesquisadores com experiência de supervisão de outros pesquisadores que utilizam pesquisa qualitativa, além de profissionais envolvidos no desenvolvimento do campo de estudo em administração (tais como coordenadores de programas de doutorado). A partir dos resultados das entrevistas, os autores identificaram habilidades, conhecimentos e práticas necessárias à condução de pesquisa qualitativa em administração e analisaram os processos de aprendizagem envolvidos em sua aquisição. Entre as habilidades (o know-how procedimental) necessárias à produção de pesquisa qualitativa de boa qualidade, os autores apontaram: a coleta e a análise de dados eficazes, a redação persuasiva, bem como a análise e a avaliação eficazes.

Quanto aos conhecimentos relativos à produção acadêmica e outros tipos de informações necessárias à compreensão do contexto de pesquisa, Cassell et al (2009), identificaram: o conhecimento do conjunto de técnicas disponíveis, o domínio das abordagens filosóficas existentes e a compreensão da complexidade inerente à pesquisa qualitativa. As atividades dinâmicas relacionadas à aquisição de competências no processo de pesquisa são definidas pelos autores como: reflexão (uso de estratégias de resolução de problemas visando gerar algum tipo de aprendizagem que possa servir de referência para ações futuras), reflexividade (avaliação crítica das suposições que o pesquisador assume como certas e inquestionáveis, em relação à pesquisa e ao seu próprio papel na pesquisa) e phronesis (termo retirado de Aristóteles, referente à "sabedoria prática", à capacidade de responder de maneira flexível e apropriada às características do contexto).

\section{Aprendizagem em organizações}

De acordo com DeFillippi e Ornstein (2003), há cinco perspectivas psicológicas subjacentes às teorias de aprendizagem organizacional (biológica, comportamentalista, cognitiva, sociocultural e psicodinâmica). A mais adotada em pesquisas sobre aprendizagem em organizações é a abordagem cognitiva. Esses autores afirmam que essas teorias psicológicas são utilizadas metaforicamente em teorias sobre aprendizagem organizacional. Este estudo adota abordagens cognitivas de aprendizagem individual e socioculturais de aprendizagem coletiva em organizações, tendo os grupos de pesquisa sido aqui considerados unidades sociais em nível organizacional. 
Segundo Abbad e Borges-Andrade (2004), de acordo com abordagens cognitivas e comportamentalistas, aprendizagem refere-se a mudanças ocorridas no comportamento (repertório de conhecimentos, habilidades e atitudes) de indivíduos em consequiência de suas interações com outras pessoas e com o ambiente externo. Entretanto, para as abordagens cognitivas, essas mudanças envolvem processos individuais de aquisição (atenção, percepção e codificação), retenção ou memorização, generalização e transferência de aprendizagem (ABBAD; BORGES-FERREIRA; NOGUEIRA, 2006).

Além desses processos cognitivos individuais, há outros que ocorrem nas organizações em níveis mais abrangentes de análise: intermediários (grupos) e mais amplos (organização e ambiente). Esses processos requerem socialização, codificação, disseminação, compartilhamento de conhecimentos, emergindo nas organizações por mecanismos de transferência horizontal ou paralela (dentro do mesmo nível de análise: entre indivíduos de um mesmo grupo, entre grupos de uma ou mais organizações ou entre organizações) e vertical (entre níveis diferentes de análise, em dois sentidos bottom-up - indivíduo/grupo/organização e top-down organização/grupo/indivíduo). Esses mecanismos são descritos por Kozlowski et al (2000), ao sugerirem uma abordagem multinível para o estudo de transferência e disseminação de aprendizagens em organizações.

A organização é vista como uma entidade que aprende, codifica, memoriza, transfere, aplica e transmite conhecimentos declarativos e procedimentais, habilidades de solução de problemas, valores, crenças e atitudes. Estudo de Akgün, Lynn e Byrne (2003) examina a aprendizagem organizacional a partir da perspectiva da cognição social, segundo a qual o processo de aprendizagem em organizações é desenvolvido por meio de redes de interações individuais e de estruturas culturais da própria organização. De acordo com esse tipo de abordagem, a aprendizagem ocorre no nível dos indivíduos e, ao mesmo tempo, no dos grupos, em virtude de suas normas, valores, rotinas e cultura organizacionais - as quais foram desenvolvidas pela aprendizagem e pelas ações de seus membros. Dessa forma, a cognição social ajuda a explicar as relações recíprocas entre aprendizagem individual e organizacional de maneira dinâmica e satisfatória.

Para Akgün, Lynn e Byrne (2003), representantes da abordagem sociocognitiva, a aprendizagem organizacional é um processo multifacetado e multidimensional e que ocorre a partir de interações recíprocas, interligadas e mediadas pela cultura organizacional. De acordo com esses autores, são 10 os componentes da cognição social - os quais, a despeito de serem correlacionados, são distintos. Esses 10 construtos sociocognitivos e suas respectivas definições estão apresentados no quadro 1.

Para Tacla e Figueiredo (2003) a aquisição e a conversão de conhecimento tácito em explícito em organizações ocorrem por meio de atividades e processos organizacionais. A aquisição externa caracteriza-se por esforços como contratação de especialistas, treinamento externo, participação em seminários e contratação de consultores. A aquisição interna se dá por meio de pesquisa e desenvolvimento e da experiência de se aprender fazendo, entre outras estratégias de aprendizagem. A conversão de conhecimento ocorre através da socialização (por exemplo: solução compartilhada de problemas, visitas de pessoas a outras organizações e criação de sistemas de disseminação da informação) e da codificação (por exemplo: elaboração de procedimentos, certificações e especificação de materiais). Observa-se que alguns nomes dos conceitos adotados nessa abordagem de aprendizagem em organizações são iguais aos adotados em teorias de aprendizagem individual (aquisição, codificação). Porém, em abordagens de aprendizagem organizacional, esses conceitos fazem 
referência a processos e atividades distintos, pertencentes a diferentes níveis de análise (grupos, unidades ou organização).

Quadro 1 - Construtos sociocognitivos da aprendizagem organizacional

\begin{tabular}{|c|c|}
\hline Conceitos & Definições \\
\hline Aquisição & Coleção de informações primárias ou secundárias de fontes variadas \\
\hline Implementação & Aplicação de informações técnicas e mercadológicas \\
\hline Disseminação & $\begin{array}{l}\text { Processo pelo qual informações de diferentes fontes são distribuídas e } \\
\text { compartilhadas }\end{array}$ \\
\hline Pensamento & $\begin{array}{l}\text { Ações intencionais, fundamentadas e direcionadas a um objetivo, envolvendo } \\
\text { resolução de problemas, formulação de inferências, cálculo de probabilidades e } \\
\text { tomada de decisões }\end{array}$ \\
\hline "Desaprendizado" & Processo de redução ou eliminação de hábitos e conhecimentos preexistentes \\
\hline Improvisação & Planejamento e implementação simultâneos de alguma ação \\
\hline Emoções & $\begin{array}{l}\text { Tendência a respostas adaptativas comportamentais e fisiológicas suscitadas } \\
\text { diretamente por situações evolucionárias significativas }\end{array}$ \\
\hline $\begin{array}{l}\text { Produção de } \\
\text { sentido } \\
\text { (sensemaking) }\end{array}$ & Atribuição de sentido a dados e informação \\
\hline Memória & $\begin{array}{l}\text { Informação armazenada (ou seja, decisões anteriores detalhadas, resultados, } \\
\text { surpresas ocorridas e as respostas produzidas pela organização, além de decisões } \\
\text { não registradas por escrito) sobre a história de uma organização }\end{array}$ \\
\hline Inteligência & $\begin{array}{l}\text { Capacidade e habilidade de processar, interpretar, manipular e usar informação na } \\
\text { organização }\end{array}$ \\
\hline
\end{tabular}

Fonte: Akgün, Lynn e Byrne (2003).

De acordo com DeFillippi e Ornstein (2003), entre as abordagens socioconstrutivistas, há dois modelos predominantes, o modelo referente a comunidades de prática e o modelo de criação de conhecimento (NONAKA; TAKEUCHI, 1995). O primeiro sugere que a aprendizagem surge dentro de comunidades de prática, nas quais os integrantes compartilham uma linguagem comum, valores e práticas. De acordo com essa abordagem, o neófito adquire, ao ingressar no grupo, uma visão de mundo e uma memória coletivas. Nesse tipo de unidade social, a aprendizagem é predominantemente informal e baseada na prática de atividades comuns. O segundo modelo, o Seci (socialização, externalização, combinação e internalização) enfatiza a natureza social da aprendizagem em organizações. Os processos abordados por esse modelo pertencem ao nível coletivo, às interações sociais e a competências de trabalho em grupo. A socialização refere-se ao compartilhamento de conhecimentos através de interações diretas entre pessoas; a externalização relaciona-se à conversão de conhecimentos tácitos em explícitos por meio de metáforas, analogias ou narrativas; a combinação diz respeito aos processos de coleta, compilação, edição e disseminação de conhecimentos explícitos e a internalização é um conceito relacionado à incorporação de conhecimentos explícitos na aplicação prática e em ações que 
favorecem o aprender fazendo e a experimentação. A descrição desses processos sociais facilita a compreensão da aprendizagem no nível de grupos e organizações, pois as definições referem-se diretamente aos níveis coletivos de análise.

Além da aprendizagem de competências coletivas relacionadas à realização cooperativa e coordenada de atividades, há aprendizagens individuais de competências humanas, definidas por meio de seus componentes (conhecimentos, habilidades e atitudes). De acordo com Abbad e Borges-Andrade (2004), conhecimento é um tipo de resultado da aprendizagem que se refere à aquisição e à compreensão de informações verbais, declarativas (definições, nomes, características de objetos, componentes de teorias) e de conhecimentos procedimentais (etapas de uma atividade, métodos). Essas habilidades intelectuais são básicas e agem como pré-requisitos para novas aprendizagens. De acordo com sistemas de classificação de resultados de aprendizagem, além dessas, existem habilidades intelectuais mais complexas, relacionadas à solução de problemas (análise, síntese ou criação e avaliação) e a estratégias cognitivas (automonitoramento, autoavaliação, controle da atenção). Neste caso, são mais difíceis de se adquirir, pois requerem estratégias de aplicação prática, aplicação de regras e normas complexas, discussão e reflexão em grupo e exposição das pessoas a situações variadas, para generalização das aprendizagens.

Segundo Abbad e Borges-Andrade (2004), há duas formas de aprendizagem em organizações: a formal e a informal ou espontânea. No primeiro caso, a organização planeja situações visando produzir resultados específicos de aprendizagem como: pela escolha de disciplinas a serem ensinadas, treinamentos, cursos e seminários. Quanto à aprendizagem informal ou espontânea, esta parte da iniciativa das pessoas, de suas observações em conversas informais no ambiente de trabalho, bem como de pedidos de auxílio ou da consulta a materiais escritos. Em grupos de pesquisas, observa-se a adoção dos dois tipos, com possível predomínio da aprendizagem informal, verificada tanto pela transmissão de conhecimentos dos mais experientes para os menos experientes, quanto pela solução compartilhada de problemas.

\section{Conhecimento: compartilhamento, armazenamento e memória}

Anand, Glick e Manz (2002, p.59) definem conhecimento organizacional como "qualquer informação, crença ou capacitação que a organização possa aplicar às suas atividades".

A criação, o armazenamento e a disseminação de conhecimentos têm sido estudados por vários autores (NONAKA; KROGH; VOEPEL, 2006), os quais, de modo geral, destacam que a gestão do conhecimento envolve a criação de meios para captação, manutenção, armazenagem, divulgação e aplicação do conhecimento na realidade organizacional. É possível, portanto, afirmar que a criação de conhecimento e a aprendizagem são vistos como processos interdependentes e complementares.

A armazenagem visa reduzir a perda de conhecimentos, contribuindo para a formação da memória coletiva, além do que, permite viabilizar o acesso a conhecimentos existentes. Para alcançar esse objetivo, usualmente, é providenciada a formação de repositórios de conhecimentos, o que exige que o conhecimento seja codificado. A transferência, compreendida como disseminação de conhecimentos, por sua vez, está associada diretamente à memória organizacional, sendo comum no dia a dia das organizações, quando funcionários conversam, 
trocando informações, e compartilham experiências. A transferência horizontal de conhecimentos, além de garantir que o conhecimento individual seja transmitido e passe a ser acessível para os demais integrantes de um grupo.

Quinello (2006) analisou o conceito de memória organizacional e apresentou algumas definições, entre as quais a que define memória como o poder ou o processo de reprodução ou resgate daquilo que tem sido aprendido e retido na organização, principalmente, por meio de três mecanismos associativos, a saber: (1) a memória episódica, que reflete o conhecimento adquirido por indivíduos; (2) a memória semântica, relacionada a conhecimentos factuais; e (3) a memória procedural, referente à retenção de etapas e processos de trabalho. Este último tipo é encontrado em manuais de instruções que ensinam como fazer coisas.

As organizações adotam sistemas para armazenar experiências individuais e coletivas, constantemente requeridas pelo trabalho. Memorizar (reter, guardar e disponibilizar) conhecimentos, em contextos organizacionais, é uma estratégia para facilitar a antecipação e a solução de problemas, o aprimoramento da tomada de decisão, o compartilhamento de conhecimentos entre pessoas e grupos, a redução da dependência da organização em relação a indivíduos e a socialização de novos integrantes. A gestão do conhecimento, calcada nas novas tecnologias da informação e comunicação, pode criar e disponibilizar não apenas repositórios de conhecimentos (declarativos e procedimentais), mas também objetos de aprendizagem interativos (como instruções, casotecas) que dão suporte à aprendizagem individual e coletiva. Além disso, facilita a manutenção e a transmissão da memória coletiva. A seguir, são descritas as estratégias metodológicas adotadas neste estudo, escolhidas a partir dos referenciais teóricos apresentados anteriormente.

\section{Método}

Esta pesquisa caracteriza-se como exploratória (RICHARDSON, 1994) e de campo, tendo empregado técnicas qualitativas: análise documental e entrevistas individuais. Exploratória, levando em conta que não foi identificado na literatura revisada um conjunto consolidado de conhecimentos a respeito dos temas abordados. De campo, pois foram coletados dados com coordenadores de 10 grupos de pesquisa de uma universidade.

$\mathrm{Na}$ universidade pesquisada existem 324 grupos validados pela instituição no $\mathrm{CNPq}$, os quais estão classificados em oito áreas de conhecimento. Para este estudo foram sorteados três grupos de cada área, perfazendo um total de 24 grupos de pesquisa. No entanto, apenas 10 líderes responderam ao convite para participar.

Os grupos respondentes foram identificados por letras e números. Pertencem às seguintes áreas: ciências agrárias (G2, G3 e G4), ciências sociais aplicadas (G6 e G7), ciências biológicas (G1), ciências da saúde (G5), ciências exatas e da terra (G11), engenharias (G9) e, por fim, linguística, letras e artes (G8).

Em cada grupo de pesquisa foi entrevistado o coordenador ou um dos coordenadores, quando havia mais de um. Dos grupos pesquisados, seis possuem mais de um coordenador. A quantidade de membros varia de oito a 104, com seis grupos reunindo mais de 20 participantes. A faixa etária dos respondentes varia de 33 a 64 anos, mas apenas três têm menos de 40 anos. Ainda em relação aos respondentes, seis possuem pós-doutorado, 
enquanto os demais são doutores. Quanto aos coordenadores, estes têm entre 11 e 34 anos de atuação em pesquisa.

As entrevistas individuais foram realizadas durante outubro e novembro de 2009, com roteiro semiestruturado, desenvolvido a partir da revisão de literatura e dos objetivos da pesquisa. Foram abordados:

- os objetivos do grupo,

- $\quad$ o processo de pesquisa e produção de conhecimento em sua área de atuação,

- $\quad$ o surgimento, desenvolvimento e funcionamento do grupo,

- $\quad$ os fatores que contribuíram para o desenvolvimento e consolidação do grupo,

- $\quad$ o ingresso de novos participantes,

- $\quad$ os pré-requisitos e as competências necessários para a participação no grupo,

- $\quad$ as principais dificuldades enfrentadas,

- $\quad$ os resultados alcançados ao longo do tempo,

- $\quad$ os aspectos e características que contribuíram positivamente para esses resultados,

- as interações e semelhanças com outros grupos de pesquisa da mesma área,

- $\quad$ o apoio dado pela universidade e pelos órgãos de fomento,

- $\quad$ as condições ambientais e materiais disponíveis,

- os procedimentos adotados para manter a memória do grupo e para a transmissão de conhecimentos internamente ao grupo,

- as mudanças ocorridas no repertório de conhecimentos, habilidades e atitudes a partir da participação no grupo de pesquisa; e

- $\quad$ o modo como essas mudanças ocorreram.

As entrevistas foram gravadas, transcritas, organizadas e analisadas, tanto individualmente quanto em seu conjunto. Eventuais trechos transcritos, para ilustrar os resultados alcançados, estão identificados de acordo com o grupo a que se referem. As categorias de análise foram definidas a posteriori, com base nos dados empíricos obtidos. Os conteúdos verbalizados foram analisados por meio do agrupamento de respostas com sentido semelhante que representassem o mesmo significado. As categorias de conteúdo foram extraídas pelos pesquisadores, que discutiram, analisaram e classificaram as respostas dos entrevistados a cada questão, buscando identificar semelhanças e diferenças nos conteúdos. Após a análise de conteúdo, foi realizada a contagem de respostas em cada uma das categorias de conteúdo. 


\section{Resultados}

Inicialmente, estão apresentados os objetivos, as características e o modo de funcionamento do grupo; em seguida, o processo de ingresso e as competências necessárias aos pesquisadores, as competências desenvolvidas e os processos de aprendizagem ocorridos com a participação nos grupos.

\section{Caracterização e modo de funcionamento dos grupos de pesquisa}

Os objetivos dos grupos de pesquisa estão relacionados à produção de conhecimento, à formação de profissionais e à integração de pessoas. Para nove dos 10 líderes entrevistados, um dos objetivos é a produção de conhecimento ou de produtos. Em um dos casos, o resultado da atuação do grupo é o estabelecimento de procedimentos para atuação (para diagnóstico, tratamento e acompanhamento de resultados) relativos ao assunto estudado.

A integração das pessoas para viabilizar o desenvolvimento de estudos num tema comum e para o compartilhamento de experiências entre os integrantes do grupo foi citada por três dos pesquisados. Em relação à formação dos profissionais, foi ressaltado, além de conhecimentos técnicos, o preparo para profissionais para a liderança no campo intelectual de atuação e o preparo quanto a comportamentos e atitudes necessários ao exercício das atividades de pesquisa.

Quanto ao modo como ocorrem a produção de conhecimento e o processo de pesquisa dentro do grupo, oito dos líderes ressaltaram o planejamento e a execução de atividades de pesquisa, quais sejam: a elaboração de um projeto de pesquisa (envolvendo a identificação de lacunas de conhecimento) e o desenvolvimento das ações planejadas, com a coleta, análise e discussão de dados, tendo sido destacado por um dos líderes a importância da reflexão quanto às ações desenvolvidas e aos resultados alcançados. Em segundo lugar, cinco líderes citaram que a produção de conhecimento ocorre a partir da interação entre pesquisadores mais e menos experientes. No que se refere à interação com outros pesquisadores, foi apontada como importante a participação em congressos, enquanto três dos líderes consideraram importante a elaboração de relatos de pesquisa especialmente, artigos apresentados em congressos e publicados em periódicos. No que diz respeito aos processos de coleta e de análise de dados, foram identificadas especificidades conforme o objeto de pesquisa de cada grupo.

Entre as técnicas de coleta de dados foram citados: observação, diário de campo, fotografias, análise de imagens, aplicação de questionários, entrevistas e a realização de experimentos em campo ou em laboratórios. A coleta de dados secundários - em bibliotecas, museus, centros de documentação e a partir de referenciais teóricos - é utilizada por um dos grupos.

O uso de abordagens quantitativas e qualitativas variadas foi a técnica empregada para a análise de dados. Isto é, foram realizados protocolos para o exame de características dos materiais coletados (genéticas, morfológicas, laboratoriais etc.), análises de conteúdo, análises estatísticas, análises históricas e culturais e análise de impacto de indicadores em sistema de produção/econômico, entre outros procedimentos adotados.

Alguns líderes destacaram a importância dos participantes do grupo terem acesso aos conhecimentos existentes, não apenas através da leitura de bibliografia básica, de artigos científicos e de livros relativos ao campo 
conceitual e a metodologias relacionadas ao tema, mas também pela interação com profissionais de áreas de pesquisa afins e pela aplicação prática de conhecimentos e habilidades de pesquisa.

Foram apresentados seis motivos para a formação dos grupos: viabilizar e/ou ampliar pesquisas, reunir pesquisadores com interesses semelhantes, atender a demandas internas e externas ao grupo, viabilizar a obtenção de recursos, atender a interesses pessoais e adquirir novas aprendizagens. Quanto à viabilização de pesquisas, foi apontada não apenas a possibilidade de obtenção de recursos financeiros e materiais nos órgãos de fomento, mas principalmente a união de esforços para viabilizar a produção de conhecimentos.

Os dados coletados indicaram uma diversidade quanto ao modo de funcionamento dos grupos, tanto no que diz respeito à periodicidade de encontros, como também ao modo de interação. Quatro grupos realizam reuniões mensais, quinzenais ou semanais, dependendo da demanda de trabalho; dois grupos comunicam-se somente por e-mail ou realizam reuniões esporádicas e três grupos realizam encontros presenciais diários nos respectivos laboratórios.

Quanto ao modo de interação, nos grupos em que a convivência é diária, ocorrem frequentes encontros entre todos os integrantes. Em outros grupos, os contatos presenciais ocorrem, principalmente, entre o orientador líder do grupo e cada um de seus orientados, sendo, em alguns casos, a comunicação feita predominantemente por meio eletrônico. Há grupos que realizam seminários periódicos em que os pesquisadores apresentam o andamento dos seus projetos, abordando resultados de coletas de dados, análises feitas e eventuais dificuldades enfrentadas durante a pesquisa. Nesses encontros, o objetivo é compartilhar o conhecimento produzido e discutir os resultados das pesquisas, cabendo observar que tais reuniões também possibilitam um acompanhamento do cronograma de atividades. Outros grupos, por sua vez, têm uma dinâmica diferente. Neles, a interação é mais individualizada, com as discussões ocorrendo entre orientador e orientado, numa periodicidade que varia de grupo para grupo e, em alguns casos, conforme as demandas do orientado e/ou do orientador.

Em alguns grupos o contato entre os integrantes é diário, uma vez que a coleta de dados é feita pelo grupo e a análise do material coletado é realizada em laboratório. Isso permite uma maior interação entre os integrantes e que dúvidas, dificuldades e soluções encontradas sejam compartilhadas cotidianamente. Em um dos grupos, a dinâmica de funcionamento é um pouco diferenciada, por tratar-se de um grupo da área de saúde que, além de pesquisas, realiza atendimento clínico.

De acordo com estudos analisados por Bastos, Gondim e Loiola (2004), são necessárias comunicação e integração interpessoal para que a aprendizagem individual atinja o nível coletivo. Diante disso, e da noção de que o conhecimento tácito exige interações mais intensas (ANAND; GLICK; MANZ, 2002), entende-se que os grupos de pesquisa que interagem e promovem reuniões com maior frequência estejam mais aptos a compartilhar conhecimentos e experiências aprendidos.

A líder do G2 ressaltou que o funcionamento do grupo depende, principalmente, do modo como é feita a gestão e a cobrança das atividades a serem realizadas pelos inúmeros integrantes. É possível que essa gestão seja mais necessária pelo fato do grupo ser disperso fisicamente. Para ilustrar o modo de funcionamento dos grupos, segue citação: 
Discute-se sobre o andamento das pesquisas, sobre perspectivas de pesquisas futuras e sobre futuras publicações. As decisões são tomadas em conjunto pelos professores. (G2)

A atuação de estudantes em grupos de pesquisas se dá não apenas pela realização de pesquisas, mas também pela participação em atividades acadêmicas internas (como leituras, reuniões ou seminários), em congressos e eventos científicos e pela redação de projetos e textos científicos.

O ingresso de novos participantes, de acordo com nove entrevistados, ocorre por meio de procedimentos formais de seleção, tais como entrevista para avaliação de interesses e afinidades com a linha e os procedimentos de pesquisa, processo seletivo da pós-graduação ou participação probatória nas atividades do grupo. Um dos líderes informou que "o potencial integrante atua por um semestre no grupo para ser avaliado" (G1).

Por sua vez, oito entrevistados citaram como critérios de seleção: interesse, tempo disponível, ter cursado matérias com os coordenadores, rendimento acadêmico e desenvolvimento de projetos afins. Há também procedimentos informais de seleção, citados por quatro entrevistados, tais como o convite de integrantes ou do próprio coordenador do grupo.

Para cinco entrevistados, são necessários conhecimentos e habilidades intelectuais, tendo sido citados, entre outros exemplos, a realização de trabalho de campo, o levantamento bibliográfico e a redação de relatórios. Alguns extratos são apresentados a seguir:

\section{Tem que saber escrever. (G8)}

Eu vejo assim: uma pessoa que já tenha feito algum trabalho de campo, algum levantamento de bibliografia e que tenha alguma disponibilidade de tempo para essas nossa duas quintas-feiras por mês. (G6)

Segundo dois entrevistados, o ingresso em seu grupo está condicionado à análise de currículo, relativa a publicações, pesquisas anteriores e formação específica (G4 e G9).

Entre os fatores que contribuíram para o desenvolvimento e a consolidação do grupo de pesquisa, oito dos respondentes apontaram interesse e motivação. A fala de dois desses líderes ilustra bem esse ponto de vista:

O esforço dos integrantes do grupo de pesquisa é a principal força de sustentação do grupo. (G1)

A cooperação e a interação da equipe é [sic] o maior fator que mantém o grupo funcionando. (G3)

Na visão de cinco entrevistados, o apoio institucional foi um dos fatores que mais contribuíram para a formação e a consolidação do grupo de pesquisa. As principais dificuldades enfrentadas ao longo da existência do grupo estariam relacionadas à falta de recursos (materiais e financeiros) e de apoio institucional (apontado por oito 
líderes), como: sobrecarga de trabalho, rotatividade de integrantes, falta de incentivo a atividades de pesquisa e falta de integração entre as atividades de ensino e de pesquisa na instituição de ensino superior.

Dificuldades envolvendo a equipe foram citadas por cinco entrevistados, tais como a pequena quantidade de pesquisadores, além do desafio da integração entre os membros do grupo (tanto pesquisadores, quanto pessoal de apoio), como ilustrado pelos trechos a seguir:

Um grupo de pesquisa deve ter pessoas formadas para ensinar aos outros. $O$ grande problema é que quando o aluno fica pronto, ele vai embora. (G1)

Outros problemas são problemas administrativos, dentro da própria universidade, [...] a gente às vezes é mal visto porque as pessoas que trabalham no nosso setor são bastante exigidas. A gente cobra muito, inclusive, dos funcionários do setor, que sabem que têm que trabalhar muito. Enquanto em outros setores, os funcionários não têm que trabalhar tanto. (G2)

As agendas, pois nós sempre estamos com as agendas sobrecarregadas. (G6)

Três entrevistados apontaram problemas decorrentes da falta de políticas de integração entre os diversos pesquisadores. Dois deles disseram o seguinte a esse respeito:

Falta uma política estruturante do país para a pesquisa, assim como um rumo por parte da universidade. Há pouca interação entre docentes. [...] Não há apoio do governo para a manutenção de redes de pesquisas internacionais. (G1)

A universidade devia estar atenta para a falta de política de pesquisa, porque eu acho que, hoje, para fazer pesquisa, é necessário envolver todas as áreas, todos os setores. (G5)

Quanto aos resultados alcançados pelo grupo ao longo do tempo, nove entrevistados mencionaram a produção e a divulgação de conhecimentos. Cinco entrevistados citaram a busca de reconhecimento e visibilidade, nacional e internacional, segundo os trechos:

Então, a gente já tem um reconhecimento sobre quais recursos genéticos são importantes e porque são importantes. [...] Antes da formação do grupo, a minha área era muito mal vista, por ser considerada muito política e [haver] pouco dado de pesquisa. (G2)

Tem gerado patentes, tem gerado visibilidade nacional; o pessoal do [jornal local] aparece aqui para fazer entrevista com os professores, tem impacto relevante. (G9)

A formação de mestres e doutores também foi considerada por três dos entrevistados um importante resultado das atividades do grupo. 
Entre os aspectos que contribuíram positivamente para os resultados alcançados pelos grupos, oito entrevistados se referiram à qualidade do trabalho em equipe, ao apoio institucional, à participação em congressos e à formação de redes interinstitucionais de pesquisa. Os trechos a seguir ilustram as respostas dadas:

O que é mais importante são as pessoas. Assim, item número um: as pessoas. Se você não tem as pessoas certas, nada acontece. (G2)

Professores com bom relacionamento é uma coisa extremamente importante, que não tem como agregar. (G9)

Rede. Sozinho ninguém faz nada. (G3)

O fato de o grupo estimular a participação de pessoas em eventos. (G6)

Em relação ao apoio institucional é possível perceber que existem diferentes situações na universidade, uma vez que parte dos pesquisados têm um opinião divergente quanto a esse apoio, como indicado anteriormente.

Quanto aos apoios recebidos da universidade ou de órgãos de fomento, há líderes que destacam o fato de receberem pouco ou nenhum apoio da universidade. Um deles reconhece que esse apoio aumentou no decorrer do tempo, mas que precisa ser maior.

Os apoios mencionados relacionam-se à disponibilização de espaço físico, laboratórios, alguns equipamentos e materiais, tempo para pesquisa, bolsas de estudos e repasse de orçamento. Já o apoio dado pelos órgãos de fomento concentra-se na concessão de bolsas de estudos e recursos a partir da aprovação de projetos de pesquisa. Os órgãos citados foram CNPq, Capes, Finatec, Finep e FAP. Dois líderes comentaram ter pouco apoio e um líder não respondeu a respeito. Não foi identificada relação entre o apoio dado pelos órgãos de fomento e o tempo de existência do grupo.

As condições ambientais (ambiente e espaço físico) e materiais (equipamentos, laboratórios etc.) nem sempre são suficientes para as atividades do grupo. É importante notar que quatro líderes disseram não dispor de condições ambientais e materiais adequados à pesquisa.

Ao comparar a interação entre grupos de pesquisa, observou-se que ela ocorre tanto com grupos internos quanto externos à universidade a qual se está vinculado. Isso foi confirmado por três líderes, enquanto outros três disseram que só há interação com grupos externos.

Os principais motivos para as interações estão relacionados ao compartilhamento de espaço físico, de equipamentos e de experimentos. Apenas um líder afirma que há interação mínima com outros grupos, sugerindo que deveria haver projeto integrado entre laboratórios distintos. Somente um grupo não mantém interação com outros grupos, segundo relato do líder, o qual observa que existem apenas vínculos individuais e esporádicos. 
Dois líderes (G2 e G6) destacaram estar em constante alerta para identificarem eventuais interações com outros grupos de pesquisa que contribuam para atender a demandas de conteúdos interdisciplinares relacionados aos temas e problemas de pesquisa com os quais se deparam no cotidiano.

Os relatos dos líderes apontam que, em geral, os grupos da mesma área funcionam de modo semelhante. Os líderes que relataram não haver semelhança indicaram as especificidades da área de pesquisa como justificativa. As principais semelhanças mencionadas abordam os objetivos do estudo, o desenvolvimento dos trabalhos e a operacionalidade dos grupos. As principais diferenças mencionadas são: a seleção dos participantes, o funcionamento dos grupos, o tamanho do grupo (quanto maior o grupo, maior a produção) e a quantidade de trabalhos orientados, de pesquisas em desenvolvimento. Os grupos da mesma área também diferem quanto ao tempo de existência do grupo. Nesse sentido, os mais antigos dispõem de maior quantidade e variedade de recursos de apoio à pesquisa, além de melhores condições de trabalho. Além disso, dentro da mesma área, há grupos que realizam pesquisas básicas e outros, pesquisas aplicadas.

\section{Compartilhamento, armazenamento e acúmulo de conhecimento}

Foi possível identificar duas maneiras pelas quais os resultados alcançados são retidos e perpetuados no grupo e como a memória do grupo é mantida: por transmissão oral e/ou por consultas a documentos e repositórios virtuais de conhecimento.

Entre os 10 líderes entrevistados, seis mostraram haver a coexistência das duas categorias em seus grupos. Isso pode ficar mais claro a partir do seguinte trecho de entrevista:

A maneira (de reter os resultados e repassá-los aos integrantes) é através dos próprios professores mais antigos, pessoalmente, e através dos artigos (por eles indicados). Tanto para professores, quanto para alunos. (G9)

O trecho em questão mostra que a transmissão oral é feita por integrantes mais experientes para os menos experientes e menos graduados. Além disso, os resultados são retidos de forma a constituir parte da memória do grupo a partir da publicação de artigos, nos quais são apresentadas as pesquisas e os resultados alcançados. Os trechos de entrevistas transcritos a seguir ilustram as respostas dadas pelos líderes:

Os alunos solicitam informações e os professores ou membros do grupo indicam artigos publicados ou lidos, revistas, apresentações em congressos, etc. (G7)

Através de oferecer aquilo que já foi publicado, como trabalhos, aos alunos, além da transmissão de conhecimento oral. (G5)

Por meio de leitura de papers, de trabalhos publicados. (G1)

\section{Aprendizagem e competências}


No que concerne à questão "O que você aprendeu com a participação no grupo? Que mudanças ocorreram em seu repertório de conhecimentos, habilidades, atitudes e valores a partir da participação no grupo de pesquisa?", foram encontrados conteúdos relativos às categorias atitudes, valores e habilidades intelectuais. Entre os 10 líderes entrevistados, um citou ter adquirido habilidades intelectuais, quatro deles disseram terem ocorrido mudanças em suas atitudes e em seus valores e cinco indicaram ter aprendido algo diferente, tanto em relação a atitudes e valores, quanto a habilidades intelectuais. Levando em consideração as passagens de entrevistas a seguir, é possível entender melhor como as respostas que abrangem as duas categorias apontam os líderes desses cinco grupos como os que mais aprenderam ou mudaram seus métodos a partir do grupo de pesquisa:

A gente aprende muito, conhecimento vem com o tempo, mas a importância, o que faz maior diferença, é aprender a valorizar quem são as pessoas. (G3)

Mudanças com interação com outros membros do grupo: conhecimentos diferentes, técnicas diferentes. E desenvolvimento de muita, muita paciência. (G9)

Então, eu aprendi [...], principalmente, a parte de ética dentro da divulgação de resultados. G4

Para outro líder, a aprendizagem no grupo de pesquisa é constante e transformadora, pois desenvolve:

\section{[...] o sentido de como conviver num grupo, como lidar melhor com o aluno, como aprender e estimular um aluno. G5}

Outra atitude, de respeito às diferenças individuais, aparece claramente na resposta do líder do G11, que diz ter aprendido no grupo a respeitar as ideias de outras pessoas e a acreditar que todos possuem alguma competência e são capazes de dar contribuições às pesquisas, independentemente, de sua formação profissional e nível de instrução. Esse líder disse também que aprendeu a respeitar as limitações e os interesses das pessoas. Cinco líderes admitiram terem aprendido essas lições, tal como reconheceu o líder do G1:

Essas mudanças ocorreram no dia a dia e passaram a ser mais aceitas por meio da meditação, processo de autocrítica do líder. Um exercício de olhar para dentro. (G1)

O modo como o aprendizado de novas atitudes ocorreu, de acordo com os pesquisados, deu-se: a partir de reflexões individuais ou por meio de autocrítica, pelo aprendizado de habilidades intelectuais, por meio de pesquisas, leituras sobre assuntos relativos aos temas pesquisados e do contato com colegas de outros grupos e de áreas afins.

Quando perguntados acerca das atividades executadas pelos estudantes no grupo de pesquisa, alguns líderes referiram-se ao que aprendem e como aprendem esses membros no grupo. Entre as habilidades intelectuais, estão: a redação de projetos de pesquisa, a análise de artigos e textos científicos, os procedimentos de coleta, de registro, de armazenamento e de análise de dados, além da elaboração de textos científicos de divulgação (resumos para comunicações em congressos, artigos, dissertações e teses). Dois líderes descreveram o que os estudantes aprendem e como se dá o processo de ensino/aprendizagem: 
Desde desenvolvimento do projeto, de escrever o projeto em si [...] eles participam de toda a parte prática. Então, eles fazem o projeto e eu falo: "vamos definir seu tema a respeito do que você quer trabalhar". Então, eu dou sugestões também. [...] "Você vai escrever o projeto de pesquisa" [...] "Eu vou ensinar como fazer pesquisa bibliográfica". Ele tenta desenvolver. Aí, eu pego, sento com ele e vamos corrigir [...] Eu acompanho os alunos nos primeiros procedimentos [...] (G4)

Basicamente, experimentos. O aluno vai analisar o experimento, conversar comigo e se for bem sucedido, vai publicar em periódicos de alto nível e, às vezes, em congressos também. (G10)

Os estudantes aprendem em suas interações com o líder, os professores e os colegas, bem como durante a realização conjunta de pesquisas. Um dos líderes destacou a importância da participação em grupos de pesquisa para o aprendizado dos participantes, pois a realização das atividades realizadas durante uma pesquisa propicia colocar em prática conhecimentos técnicos e metodológicos, bem como interagir com pesquisadores e pesquisados. Esses tipos de estratégias de aprendizagem são denominados por Warr e Bunce (1995) e por Zerbini e Abbad (2008) de "aplicação prática" e "busca de ajuda interpessoal". O enfrentamento de dificuldades nos processos de coleta e de análise de dados, bem como o questionamento e a reflexão sobre os resultados obtidos, associados à busca de compreensão dos fenômenos estudados, foram apontados como importantes aprendizados adquiridos por estudantes em grupos de pesquisa, de acordo com alguns dos líderes.

Grande parte dos conhecimentos adquiridos pelos estudantes não se refere a conhecimentos declarativos, passíveis de serem aprendidos em exposições orais e pela leitura de textos. Os conhecimentos adquiridos por estudantes são complexos e envolvem procedimentos, métodos, técnicas, normas e teorias. São habilidades intelectuais de solução de problemas complexos, para os quais não há somente uma solução correta, mas diversos caminhos e modos de resolução. Esse tipo de habilidade intelectual, geralmente, requer a adoção de estratégias de ensino e aprendizagem voltadas à aplicação prática e à solução colaborativa de problemas, além do domínio de grande quantidade de conhecimentos declarativos e procedimentais, bem como de atitudes favoráveis ao trabalho em grupo.

\section{Conclusão}

Os grupos de pesquisa diferem entre si no que concerne aos objetos de estudo, ao tempo de existência, ao apoio institucional e à quantidade e à qualidade dos recursos de apoio recebidos de órgãos de fomento. De modo geral, os grupos foram criados para facilitar a produção de conhecimentos, reunindo pesquisadores com interesses comuns.

Os grupos consolidaram-se como resultado do esforço, do interesse e do empenho de seus integrantes, bem como do apoio material e financeiro recebido para a realização das pesquisas. Foram citadas dificuldades para se obter apoio institucional, interno e externo (dos órgãos de fomento), para a formação de redes interinstitucionais de pesquisa e intercâmbio entre universidades nacionais e estrangeiras. Foi apontada como uma restrição situacional ao desempenho dos líderes nos grupos a sobrecarga de trabalho a que estão sujeitos os 
pesquisadores, que acumulam múltiplas atividades acadêmicas além daquelas referentes à produção de conhecimentos.

Dentre os resultados mais relevantes alcançados pelos grupos, destacaram-se a produção e a divulgação de conhecimentos. A efetividade das ações do grupo é atribuída por quase todos os líderes à qualidade do trabalho em equipe. Entre os resultados obtidos ou almejados, foram citados por vários líderes, a formação de redes e a busca de reconhecimento e visibilidade (nacional e internacional) dos conhecimentos produzidos pelo grupo.

A aprendizagem nos grupos, segundo os líderes, ocorre informalmente em situações de realização conjunta de pesquisas e de discussão de resultados. Esse tipo de estratégia de aprendizagem é denominado "aprender fazendo" e "solução colaborativa de problemas". Essas estratégias coletivas de aprendizagem são similares às verificadas em comunidades de prática, mencionadas por abordagens socioconstrutivistas de aprendizagem, citadas por DeFillippi e Ornstein (2003). Os estudantes aprendem com os mais experientes por transmissão oral, por leituras dirigidas e pela solução de problemas ocorridos durante as atividades de pesquisa e a discussão de resultados. A memória do grupo parece ser transmitida, principalmente, por meio de conversas entre os membros, por relatos e pelo registro dos resultados de pesquisa em artigos e livros.

Os grupos procuram compartilhar normas e valores relacionados a abordagens e a métodos de pesquisa. Nesses casos, os integrantes mais experientes e influentes (os líderes) são os principais agentes dessa transmissão oral. $\mathrm{O}$ sentido de hierarquia, descrito pelo CNPq como característica dos grupos de pesquisa, foi claramente evidenciado nas respostas dos líderes sobre as interações sociais. A transmissão oral de valores, procedimentos, práticas, normas e padrões, tal como relatada pelos pesquisados, assemelha-se ao processo de socialização. A realização conjunta de pesquisas, por outro lado, assemelha-se aos processos de internalização de conhecimentos práticos, de acordo com o modelo Seci de Nonaka e Takeuchi (1995). Há, além destes, processos mais formais de aprendizagem, como a orientação para elaboração de projetos, feita pelo líder aos seus estudantes de mestrado e de doutorado, bem como a participação em congressos e em outros eventos científicos para a aquisição de novos conhecimentos e habilidades de pesquisa. Entre as estratégias de aprendizagem adotadas pelos grupos estão as consultas a documentos e a textos contidos em repositórios de conhecimentos, como bibliotecas virtuais. Essa estratégia de aprendizagem é denominada por Warr e Bunce (1996) de busca de ajuda em materiais escritos.

Os líderes relataram a aprendizagem de novos valores e atitudes favoráveis ao trabalho em equipe e de respeito à diversidade humana no grupo. Fazem referência a habilidades de gestão de pessoas, ligadas à coordenação de atividades, ao controle de diferentes ritmos, à valorização e ao aproveitamento das contribuições de integrantes menos instruídos. Esse tipo de competência foi também relatado por participantes de grupos de pesquisa estudados por Haythornthwaite (2006) e por Odelius e Sena (2009). Os estudantes, de acordo com a percepção dos líderes, adquirem habilidades intelectuais relacionadas à realização de pesquisas (análise de textos científicos, redação e planejamento de pesquisa, coleta, análise, interpretação de dados e elaboração de relatos de pesquisa, entre outros procedimentos). Esses resultados corroboram os encontrados por Haythornthwaite (2006) e por Odelius e Sena (2009).

Esta pesquisa contribuiu para identificar lacunas na produção de conhecimentos sobre o funcionamento e os processos de aprendizagem em grupos de pesquisa, tendo sido adotado método eficaz de análise qualitativa, 
pois encontrou resultados consistentes, que corroboram pesquisas descritivas similares. Os resultados confirmam a importância da participação de estudantes de pós-graduação e de graduação em grupos de pesquisa, possibilitando a aprendizagem de complexas habilidades intelectuais e atitudes, as quais não são comumente adquiridas em salas de aula ou em outras atividades tradicionais de ensino.

Mais estudos são necessários a respeito de grupos de pesquisas, principalmente, porque permanecem em aberto muitas questões, tais como: Grupos mais produtivos diferem dos menos produtivos em seus processos de aprendizagem? De que modo o perfil do líder afeta a qualidade das interações e a aprendizagem nos grupos de pesquisa? Como são criadas e mantidas redes interinstitucionais de pesquisa? Qual o alcance delas? Quais os conteúdos transacionados nessas redes?

Ratificando que o escopo deste estudo englobou a investigação dos líderes de grupos de pesquisa e que os demais partícipes foram tema de outro estudo, cabe observar que a ampliação desta investigação para uma mostra representativa de grupos de pesquisa tende a possibilitar um diagnóstico abrangente da situação. Isso poderia subsidiar o aperfeiçoamento de políticas de apoio a esses grupos e à formação de redes internacionais e interinstitucionais de pesquisa, além do aprimoramento da formação de estudantes de graduação e de pósgraduação.

Os resultados deste estudo suscitam uma agenda para futuras pesquisas com os seguintes tópicos:

- $\quad$ ampliação da pesquisa, uma vez elaborado e validado um questionário sobre aprendizagem em grupos de pesquisa, o qual tomaria por base as categorias de conteúdo aqui levantadas;

- comparação de diferentes grupos quanto aos seus resultados, processos de aprendizagem e qualidade das interações sociais; e

- triangulação de métodos e técnicas de coleta de dados, adotando-se medidas baseadas em percepções (questionários e entrevistas) e medidas objetivas, indicadores de produção intelectual dos membros dos grupos (análises documentais) na comparação entre grupos de pesquisa de áreas distintas.

\section{Referências bibliográficas}

ABBAD. G. S.; BORGES-ANDRADE, J. E. Aprendizagem humana em organizações de trabalho. In: ZANELLI, J. C.; BORGES-ANDRADE, J. E.; BASTOS, A. V. B. Psicologia, organizações e trabalho no Brasil. Porto Alegre: Artmed, 2004. p.237-275.

; BORGES-FERREIRA, M. F.; NOGUEIRA, R. S. F. Medidas de aprendizagem em avaliação de TD\&E. In: BORGES-ANDRADE, J. E.; ABBAD, G. S.; MOURÃO, L. (Org.). Treinamento, desenvolvimento e educação em organizações e trabalho: fundamentos para a gestão de pessoas. Porto Alegre: Artmed, 2006. p.469-488.

ACEDO, F. J. et al. Co-authorship in management and organizational studies: an empirical and network analysis. Journal of Management Studies, v.43, n.5, p.957-983, 2006. 
AKGÜN, A. E.; LYNN, G .S; BYRNE, J. C. Organizational learning: a socio-cognitive framework. Human Relations, v.56, n.7, p.839-868, 2003.

; REILLY, R. Multi-dimensionality of learning in new product development teams. European Journal of Innovation Management, v.5, n.2, p.52-72, 2002.

ANAND, V.; GLICK, W. H.; MANZ, C. C. Capital social: explorando a rede de relações da empresa. Revista de Administração de Empresas, v.42, n.4, p.57-73, 2002.

ARGOTE, L. Reflections on two views of managing learning and knowledge in organizations. Journal of Management Inquiry, v.14, n.1, p.43-48, Mar. 2005.

BASTOS, A. V. B.; GONDIM, S.; LOIOLA, E. Aprendizagem organizacional versus organizações que aprendem: características e desafios que cercam essas duas abordagens de pesquisa. Revista de Administração da Universidade de São Paulo, v.39, n.3, p.220-230, 2004.

BORGES-ANDRADE, J. E. Em busca do conceito de linha de pesquisa. Revista de Administração Contemporânea, v.7, n.2, p.157-170, 2003.

CASSELL, C. et al. Learning to be a qualitative management researcher. Management Learning, v.40, n.5, p.513-533, 2009.

CNPq - Conselho Nacional de Desenvolvimento Científico e Tecnológico. Diretório de Grupos de Pesquisa. < Disponível em: http://www.cnpq.br>. Acesso em: 28 jan. 2010.

DeFILLIPPI, R.; ORNSTEIN, S. Psychological perspectives underlying theories of organizational learning. In EASTERBYSMITH, M.; LYLES, M. A. (Ed.). Handbook of organizational learning and knowledge management. London: Blackwell Publishing, 2003. p.19-37.

DU, F. Building action research teams: a case of struggles and successes. Journal of Cases in Educational Leadership, n.2, v.12, p.8-18, 2009.

ERLEN, J. A. et al. Multiple authorship: issues and recommendations. Journal of Professional Nursing, v.13, n.4, p.262270, 1997.

FRIEDMAN, V. J.; LIPSHITZ, R.; POPPER, M. The mystification of organizational learning. Journal of Management Inquiry, v.14, n.1, p.19-30, 2005.

GLADNEY, A. P. et al. Consistency of findings produced by two multidisciplinary research teams. Sociology, v.37, n.2, 297-313, 2003.

HAYTHORNTHWAITE, C. Learning and knowledge networks in interdisciplinary collaborations. Journal of the American Society for Information Science and Technology, v.57, n.8, p.1079-1092, 2006.

HOLLIS, A. Co-authorship and the output of academic economists. Labour Economics, v.8, p.503-530, 2001.

KOZLOWSKI, S. W. J. et al. A multilevel approach to theory and research in organizations: contextual, temporal and emergent processes. In: KLEIN, K. J.; KOSLOWSKI, S. W. J. (Ed.). Multilevel theory, research, and methods in organizations: foundations, extensions and new directions. San Francisco: Jossey-Bass, 2000. p.157-210.

MAUTHNER, N. S.; DOUCET, T. A. Knowledge once divided can be hard to put together again: an epistemological critique of collaborative and team-cased research practices. Sociology, v.42, p.971-985, 2008. 
NONAKA, I.; von KROGH, G.; VOEPEL, S. Organizational knowledge creation theory: evolutionary paths and future advances. Organizational Studies, v.27, n.8, p.1179-1208, 2006.

; TAKEUCHI, H. The knowledge creating company. New York: Oxford University Press, 1995.

ODELIUS, C. C.; SENA, A. C. Atuação em grupos de pesquisa: competências e processos de aprendizagem. FACES Revista de Administração, v. 8, n. 4, p. 13-31, out./dez. 2009.

ORTENBLAD, A. A typology of the idea of learning organization. Management Learning, v.33, n.2, p.213-230, 2002.

QUINELLO, Robson. Organizational memory and forgetfulness generating vulnerabilities in complex environments. Revista de Administração Contemporânea, Curitiba, v. 10, n. spe, 2006.

RICHARDSON, L. 1994. Writing: a method of inquiry. In: DENZIN, N. K.; LINCOLN, Y. S. (Ed.). Handbook of Qualitative Research. Thousand Oaks, CA: Sage, 1994. p.516-529.

TACLA, C.; FIGUEIREDO, P. N. Processos de aprendizagem e acumulação de competências tecnológicas: evidências de uma empresa de bem de capital no Brasil. Revista de Administração Contemporânea, v.7, n.3, p.101-126, 2003.

WARR, P.; BUNCE, D. Trainee characteristics and outcomes of open learning. Personnel Psychology, 48, p.347-375, 1995.

ZERBINI, Thais; ABBAD, Gardenia da Silva. Estratégias de Aprendizagem em Curso a Distância: Validação de uma Escala. Psico-USF. , v.vol.13, p.177 - 187, 2008. 